\title{
LA IDENTIDAD LINGÜÍSTICA DEL CONTEXTO ESCOLAR
}

\author{
Autor Principal: \\ Mg. Luis HERRERA ${ }^{1}$ \\ Colaboradoras: \\ Paula ESPINOZA ${ }^{2}$ \\ Katterine FLORES ${ }^{3}$ \\ Yoselin ITURRIAGA ${ }^{4}$ \\ Javiera POBLETE ${ }^{5}$
}

\begin{abstract}
Resumen
Entendiendo la identidad lingüística como el vínculo y sentido de pertenencia de un individuo o institución con las particularidades verbales de la comunidad de habla, este artículo tiene por objetivo analizar la identidad lingüística de establecimientos escolares urbanos de la ciudad de Talca, Chile. El estudio se ha realizado desde un enfoque cualitativo entrevistando a estudiantes de 29 escuelas de distintas dependencias
\end{abstract}

\footnotetext{
${ }^{1}$ Universidad Autónoma de Chile

5 poniente, \#1670 Talca

Luis.herrera@gmail.com

${ }^{2}$ Liceo Pablo Neruda

6 oriente, \# 1240 Talca

pauandreae@gmail.com

${ }^{3}$ Liceo Juan Gomez Millas

Orilla de Maule, $\mathrm{km} / 16$ San Ignacio

Flores.lopez@gmail.com

${ }^{4}$ Sociedad Exportadora Fénix Ltda.

Avenida Ramón Freire 2990, Romeral

y.iturriagap@gmail.com

${ }^{5}$ Colegio Macchu Picchu

José Orlando Arismendi Curicó

jpoblete112@gmail.com

Fecha de recepción del artículo: Marzo 2015

Fecha de evaluación: Mayo 2015
} 
administrativas: municipales, particulares subvencionados y particulares pagados. Los resultados evidencian una identidad lingüística marcada por la asimetría entre funcionarios escolares y estudiantes, comparaciones y fomento de la competencia, que disminuyen el compromiso y sentido de pertenencia de los informantes, poniendo en riesgo el éxito escolar. Por el contrario, felicitaciones, altas expectativas y prácticas discursivas positivas generan en los estudiantes empatía con la identidad lingüística. Se sugiere reflexionar sobre la intención pedagógica de las decisiones verbales, desechando emisiones que reproducen un contexto de autoritarismo, jerarquías y competitividad.

Palabras claves: identidad, lingüística, cultura escolar, discurso, contexto.

\begin{abstract}
:
Understanding linguistic identity as the bond and sense of belonging to an individual or institution with verbal peculiarities of the speech community, this article aims to analyze the linguistic identity of urban schools of the city of Talca, Chile. The study was conducted from a qualitative approach to interviewing students from 29 schools in different administrative units: municipal, subdized and private paid. The results show a linguistic identity marked by the asymmetry between school officials and students, comparisons and promoting competition, lowering the commitment and ownership of the informants, jeopardizing school success. By contrast, congratulations, high expectations and positive discursive practices generate empathy in students with linguistic identity.It is suggested to reflect on the pedagogical intention of verbal decisions, discarding emissions that play a context of authoritarianism, hierarchy and competitiveness.
\end{abstract}

Keywords:identity, linguistics, school culture, discourse, context.

\title{
Résumé:
}

Comprendre l'identité linguistique comme le lien et le sentiment d'appartenance à un individu ou une institution avec des particularités verbales de la communauté de la parole, cet article vise à analyser l'identité linguistique des écoles urbaines de la ville de Talca, Chili.L' étude a été réalisée à partir d'une approche qualitative aux étudiants d'entrevue de 29 écoles dans différentes unités administratives: municipales, subventionné et privé payé. Les résultats montrent une identité linguistique marquée par l'asymétrie entre les autorités scolaires et les étudiants, des comparaisons et promouvoir la concurrence, l'abaissement de l'engagement et de l'appropriation des informateurs, mettant en péril la réussite scolaire. En revanche, félicitations, des attentes élevées et les pratiques discursives positifs génèrent l'empathie des élèves avec identité linguistique. Il est suggéré de réfléchir sur l'intention pédagogique de décisions verbales, en écartant les émissions qui jouent un contexte d'autoritarisme, de hiérarchie et de la compétitivité.

Mots-clés: l'identité, la langue, la culture de l'école, contexte, discours.

\section{Introducción}

Concebir todos los aspectos involucrados en un contexto escolar podría arrojar una amplia lista de factores, recursos y actores. Desde los lineamientos curriculares hasta las evaluaciones en el aula, se puede transitar por múltiples problemáticas que conciernen a la didáctica, la política, la ideología, la sociedad, el lenguaje, entre otras. De alguna manera, la intención didáctica del proceso educativo depende de la confluencia de todos 
estos factores y, en ese sentido, comprender la cultura escolar y los aspectos identitarios que la envuelven, representa una complejidad importante.

Sin lugar a dudas, todos los actores del proceso educativo co construyen la identidad y la cultura que impera en las instituciones y de alguna manera, a través de sus esfuerzos, expectativas, creencias y prácticas van generando impacto en los otros integrantes de la comunidad. De ese modo, el concepto de "escuela" o nuestra escuela" va siendo significado por cada uno de los participantes.

No obstante, cuando se ha investigado en este tema de la cultura escolar o qué "comunica" nuestro sistema educativo, nos vamos encontrando que los resultados están lejos de ser óptimos. En un estudio de la Universidad de Chile del año 2011 que indagaba en las percepciones de los estudiantes sobre los propósitos de la educación que reciben, se describió un fenómeno, por lo menos, digno de atender, pues destacaba la conclusión, entre otras, que el estudiante se educa para respetar la jerarquía y las normas, no señalándose el fomento de "la creatividad, la libertad, la autonomía y el ser un real aporte a la sociedad o, por lo menos, aquello no es percibido por los alumnos" (Herrera, 2014: 91).

Por cierto que en estas profundas interrogantes y significados que surgen de nuestro sistema educativo, el lenguaje, más que nunca, adquiere un protagonismo clave, considerando que "el intercambio de significados es un proceso interactivo: para ser intercambiados entre los miembros, los significados que constituyen el sistema social deben, en primer lugar, ser representados en alguna forma simbólica intercambiable, y la más accesible de las formas disponibles es el lenguaje" (Halliday, 1978, cit. en Lozano, Peña, Abril, 1999: 40). Más aún, si lo tomamos desde la perspectiva de Bourdieu, es decir, no como un instrumento para transmitir información, sino también como instrumento que "re-crea y actualiza relaciones de poder simbólico entre individuos y grupos que usan el lenguaje como parte de un proceso de reconocimiento y de distinción respecto de otros individuos y grupos" (Gómez, Herrera, 2014: 81). En ese sentido, al interactuar los integrantes de la cultura o la comunidad forjan actos comunicativos que dan cuenta de determinados discursos, representativos del contexto social, que terminan por definir una identidad lingüística, entendida como ese habla en común o esas decisiones lingüísticas de los individuos, que los hacen integrantes de una comunidad o grupo social determinado

Bajo estos supuestos y premisas nos ha parecido pertinente analizar la identidad lingüística de 29 establecimientos escolares urbanos de la ciudad de Talca, a través de un enfoque interpretativo, mediante la entrevista a estudiantes de séptimo y octavo año (12 a 14 años) de enseñanza básica, niveles que consideramos óptimos, pues coinciden con el momento de desarrollo en que surgen las construcciones identitarias, las crisis existenciales o sentimientos de ambivalencia.

Por supuesto que nuestro propósito no apunta a obtener conclusiones representativas que permitan generalizar a todos los contextos educacionales, pero sí a fomentar una reflexión trascendental sobre la identidad lingüística que co construyen todos los actores de una institución educativa, a partir de la información suministrada por el centro del proceso pedagógico: los estudiantes.

\author{
Marco teórico \\ Contexto, discurso y cultura escolar
}


En el tejido social que involucra a las comunidades y a la sociedad en su conjunto, se ha discutido bastante sobre las implicancias que juega el contexto, la influencia que ejerce sobre el discurso y, finalmente, en las determinaciones y modelos mentales que genera en los individuos. De una u otra manera, no se puede obviar que el contexto exterior es conceptualizado e integrado en un modelo mental de contexto que encamina e influye en las emisiones discursivas, es decir, el modelo contextual controla profundamente los procesos de producción y comprensión, pues determina "qué conocimiento general y qué información de los modelos situacionales (experiencias, noticias) expresar y presuponer en las estructuras semánticas globales y locales del discurso" (Van Dijk, 2012: 156).

En ese sentido, el discurso y el contexto de los individuos interactúan y se amalgaman en las representaciones mentales que poseen, constituyéndose, después de todo, en una parte funcional propia de los contextos globales, siendo, por cierto, una dependencia o confluencia digna de estudiarse en los análisis sociales del discurso(Van Dijk, 2008: 38).

Para ejemplificar aún más esta perspectiva teórica, bastaría detenerse en aspectos como la dominación y la desigualdad, para relacionar las intercomunicaciones entre contextos y discursos, sobre todo el cómo se retroalimentan entre sí. Es decir, la manera en que el contexto determina al discurso y, por otro lado, cómo el discurso reproduce y sustenta al contexto, subyaciendo a los ambientes ideológicos que imperan:

Las prácticas discursivas pueden tener efectos ideológicos de peso, es decir, pueden ayudar a producir y reproducir relaciones de poder desiguales entre (por ejemplo) las clases sociales, las mujeres y los hombres, las mayorías y las minorías culturales o étnicas, por medio de la manera como representan los objetos y sitúan a las personas (Fairclough\&Wodak, 2001, cit. en Araya, 2011: 15)

Si ahora, habiendo dicho lo anterior, nos centramos en lo que sucede dentro de cualquier organización humana o institución, se puede reflexionar en torno a la naturaleza de la identidad que se construye al interior de tal complejo. Por tanto, desde esa mirada, la interacción jugaría un papel clave en la construcción de dicha identidad, es decir, una manera de analizar este tema es estudiando "cómo se manifiesta la orientación de los participantes hacia sus identidades institucionales en los detalles de la conducta verbal mediante la cual conducen sus tareas institucionales" (Van Dijk, 2008: 148). En otras palabras, a partir de las prácticas discursivas o intenciones lingüísticas en la interacción de todos los actores se va construyendo la identidad institucional, siempre dentro, se insiste, de un modelo contextual, en este caso colectivo, que es la representación mental del contexto real. En definitiva, las elecciones léxicas surgen y se determinan de la mano del contexto institucional:

(...) la selección de términos descriptivos y otros ítems léxicos que los participantes consideran apropiados a (y por lo tanto indicativos de) su interpretación de la situación en la que se encuentran. (...) Lo que debe tenerse en cuenta aquí es que los hablantes se orientan hacia la institucionalidad del contexto, en parte, mediante su selección de términos entre una variedad de modos alternativos de describir a las personas, objetos o sucesos (Van Dijk, 2008: 151). 
Naturalmente, no solamente en instancias organizadas o circunstancias institucionales, también nuestras prácticas verbales cotidianas y nuestros interlocutores regulares, van constituyendo un esquema estructurado en el cual se vincula discurso y contexto. De cualquier manera, nuestras prácticas comunicativas habituales van construyendo una identidad propia en el ambiente que sea nos encontremos (Van Dijk, 2012).

Siguiendo esa línea argumental, la escuela es un fiel reflejo de lo acá discutido. Comprende una organización institucional, enmarcada dentro de un contexto, que implica determinados discursos acaecidos dentro de una rutina habitual para todos los actores, constituyéndose una cultura escolar de patrones de significado que se transmiten en el tiempo, incluyendo tradiciones, creencias, valores, normas compartidas por los integrantes de la comunidad escolar (Stolp y Smith, 1994).

Si a esa red de interacciones que da vida a la cultura escolar le sumamos las complejidades particulares de los contextos de origen de todos los individuos, nos enfrentamos a un fenómeno de una magnitud demasiado compleja si queremos lograr aprendizajes en los educandos. Por tanto, para lograr comprender al estudiante, debemos entre otras cosas, familiarizarnos con el entorno en el cual ellos se desenvuelven, absorber sus variados y múltiples conocimientos, conocer específicamente sus necesidades e intereses, su entorno familiar, puesto que, de esta manera podemos influir y llegar de otra forma hacia y hasta el alumno. En simples palabras, implicarnos en la realidad del estudiante.

Bajo ese precepto, Vigotsky es claro en señalar que para comprender al individuo, debemos entender las relaciones sociales que lleva a cabo, poniendo énfasis al origen sociocultural de los procesos psicológicos de los estudiantes (Díaz, 2009), toda vez que, en palabras de Dewey, la escuela es una institución social, en la cual se orientan los objetivos para reproducir normas, conocimientos y los procesos históricos-culturales de la sociedad (Montoya, 2007).

Entonces cobra vital importancia la interacción que tienen todos los actores de la cultura escolar y, sobre todo, el profesor con el alumno dentro de la sala de clase, cuando se comunican para lograr de esta manera aprendizajes significativos, teniendo en alta consideración, además, que "El alumno, como parte fundamental del proceso educativo, posee una serie de expectativas, intereses, motivaciones o creencias, sobre la institución escolar que deben tenerse en cuenta como elementos mediadores en el desarrollo de una tarea educativa" (Mateos, 2008: 287). De ese modo, es relevante considerar las percepciones que poseen los estudiantes sobre la institución, ya que aquello va a incidir en el rendimiento, la integración y las relaciones sociales: si posee una imagen negativa del profesorado, de sus compañeros, de sí mismo o de la organización educativa, se corre el riesgo de fracaso escolar, dificultades comunicativas o desadaptación y, en el surgimiento de tales causas, juega un papel fundamental el docente durante el proceso de enseñanza y aprendizaje (Mateos, 2008).

\section{Identidad lingüística y escuela}

Hoy en día, el concepto de identidad vive un proceso de transformación, ya que la globalización, la integración cultural y las nuevas tecnologías están mermando las fronteras históricas y conceptuales que daban cuenta de estas particularidades de un determinado grupo social. En ese sentido, ser enfático en delimitar una identidad de otra, es una tarea de suyo difícil, tanto geográfica como teóricamente, debido al: 


\title{
Centro de Estudios Interdisciplinarios en Etnolingüistica y Antropología Socio-Cultural
}

"desacuerdo en la propia terminología, su uso impreciso e incongruente, la variedad de métodos e instrumentos, la falta de unanimidad y la diversidad de elementos, categorías y/o variables que se pretenden incluir en la conformación de la identidad, la falta de sistematicidad, los reduccionismos, etc." (Agulló, 1997: 218)

No obstante, existe consenso que cuando un individuo se autoreconoce con determinadas características, conductas y actos es porque está adquiriendo el sentido de identidad (Melucci, 1982 cit. en Monereo, Pozo, 2011) y, más profundamente, cuando la identificación de tales aspectos propios coinciden con los que identifica en su entorno. Por tanto, este moldeamiento del ser en base a las asociaciones con su contexto, evidencian el papel predominante de la sociedad y específicamente del entorno social cercano en la formación de conductas y procederes de los sujetos: se construye la identidad participando en las prácticas de la comunidad y en la generalización colectiva del conocimiento (Brown \& Dyson, 2003; cit. en Monereo, Pozo, 2011):

\begin{abstract}
"Identidad es, pues, un proceso que se inicia en lo más íntimo y profundo de un individuo o de un pueblo, se traslada luego fuera de él para contactar y recibir el influjo y las percepciones del mundo y retorna entonces, internalizado, en características de hecho y de destino, de cultura y de vida, presto a dar testimonio de lo que uno es y de lo que otros perciben que uno es" (Alarcón, 1990: 3).
\end{abstract}

Dando cuenta, por cierto, de una representación mental de comunidad, que se construye con relatos, actos, símbolos que son comunes para todos los individuos que comparten dicha identidad (Monereo \& Pozo, 2011), lo cual nos hace ampliar la noción de identidad individual e insertarlo en la realidad social, enfrentándonos a una nueva concepción, la identidad cultural. Es decir, "El concepto de identidad cultural encierra un sentido de pertenencia a un grupo social con el cual se comparten rasgos culturales, como costumbres, valores y creencias. La identidad no es un concepto fijo, sino que se recrea individual y colectivamente y se alimenta continuamente de la influencia exterior" (Molano, 2008: 73).

Habiendo hecho el tránsito de identidad a identidad cultural, es evidente que el lenguaje también se implica en este fenómeno, erigiéndose el concepto de identidad lingüística, entendido como "el vínculo que crea el individuo con la comunidad de habla de la que se hace miembro y cuya variedad de lengua adopta como suya propia" (Martínez, 2008: 88). Se específica, además, que esta apropiación o, identidad lingüística no es sólo para un contexto particular, sino que mediante la capacidad de adecuación, el ser humano posee la facultad para desarrollarse social y lingüísticamente en todos los ámbitos necesarios de la vida. Más precisamente, "Un hablante no construye una sola y unificada identidad lingüística para las distintas situaciones comunicativas. Construye identidades lingüísticas múltiples y complejas" (Martínez, 2008: 88-89). Por tanto, se enfatiza que la identidad lingüística es propia de cada individuo y se va modificando de acuerdo al anillo socio-cultural y económico en el que se encuentre, de tal manera que la puede complementar con variaciones diversas si migra a otro grupo hablante. En ese sentido, es importante subrayar que la identidad lingüística del individuo responde a distintas y determinadas convivencias (Ricoeur, 1998). 
Ahora bien, retomando la óptica de una organización o institución, a partir de la perspectiva de Teun Van Dijk:

Podemos definir la organización como una colectividad social, que se produce, se reproduce y se transforma por medio de las prácticas de comunicación habituales, interdependientes y deliberadas de sus miembros. (...) Esto no quiere decir que las organizaciones sean "nada más" que discurso, sino que este es el principal medio por el cual los miembros de una organización crean una realidad social coherente que encuadra la percepción que tienen de su propia identidad (Van Dijk, 2008: 263).

Entonces, existe una relación indivisible formada, por un lado, por lo que decimos, esto es, el discurso emitido por medio del lenguaje y, por otro, la identidad. Por tanto, "un cambio en las condiciones comunicativas, provoca cambios en la identidad lingüística, es decir en la aceptación de las normas establecidas o vigentes" (Dahmen, Holtus, Kramer, Melzeltin, Schweickard,Winkelmann, 2006: 211). Ocurriendo esto dentro de una institución, sobre todo educativa, las implicancias que tienen en las prácticas verbales, las conductas y las acciones, juega un rol trascendental. En consecuencia, es necesario un discurso emitido por los establecimientos escolares que construya una identidad lingüística institucional -general a todos los individuos involucrados- que propicie un ambiente grato, cálido y significativo, en que los interlocutores transiten por un camino en común y haya una generalización de las expectativas como grupo de trabajo y no exista una parcelación de las perspectivas o percepciones negativas sobre la cultura escolar generada.

La función que tiene el lenguaje en el proceso de enseñanza-aprendizaje es primordial; de la misma forma que la relación educando-profesor. El contexto determina determinados discursos que caracterizan una identidad lingüística dentro de la cultura escolar. De tal forma, si dicha identidad lingüística "propone" decisiones verbales positivas y gratas para la convivencia de los estudiantes y todos los actores, será más efectivo el logro de los propósitos que posea el establecimiento escolar. $\mathrm{Si}$, por el contrario, la identidad lingüística está asociada a discursos negativos es más probable que el interlocutor sufra desmotivación, desinterés, rechazo, fracaso o deserción escolar, no encontrando un sentido significativo al proceso pedagógico escolar.

\section{Metodología}

Este estudio se enmarca dentro del enfoque cualitativo, no experimental y descriptivo, considerando la interioridad subjetiva del sujeto como foco de análisis. Se ha utilizado la entrevista semi estructurada en 29 estudiantes de séptimo y octavo básico, representantes de las distintas dependencias educativas del sistema escolar chileno:

10 estudiantes de establecimientos municipales(M1, M2, M3, M4, M5, M6, M7, M8, M9, M10)

15 estudiantes de establecimientos particulares subvencionados (PS1, PS2, PS3, PS4, PS5, PS6, PS7, PS8, PS9, PS10; y católicos PS11, PS12, PS13, PS14, PS15) 
4 estudiantes de establecimientos particulares (PP1, PP2, PP3, PP4)

Cabe destacar que los estudiantes fueron seleccionados a partir de un muestreo no probabilístico intencional por conveniencia.

La entrevista semi estructurada utilizada como técnica de recolección de la información, está dividida de acuerdo a los objetivos del estudio (ANEXO 1). El objetivo general es:

Analizar la identidad lingüística de los establecimientos escolares urbanos de la ciudad de Talca; los objetivos específicos, a su vez, son:

Describir la identidad lingüística de los establecimientos escolares urbanos de la ciudad de Talca Comprender las percepciones y creencias de los estudiantes acerca de la identidad lingüística de los establecimientos escolares urbanos de la ciudad de Talca.

Además, el instrumento fue validado por tres académicos con formación de pregrado en Pedagogía en Español y postgrados en gestión, curriculum y didáctica.

Por último, señalar que los entrevistados firmaron un consentimiento informado, aceptando la utilización de la información para los fines de esta investigación, resguardando el anonimato (ANEXO 2).

Una vez transcrita la entrevista, se clasificó la información en distintas categorías, según los objetivos específicos (ANEXO 3) y fueron analizados según las dependencias administrativas.

\section{Análisis de los resultados}

1. Describir la identidad lingüística de los establecimientos escolares urbanos de la ciudad de Talca

\subsection{Denominación de funcionarios}

Dentro de la totalidad de los establecimientos municipales se utiliza la denominación de "Directora" o "Director" para referirse a la autoridad máxima; como también existe la denominación de "Inspector" en todos los establecimientos de esta dependencia, incluyendo variantes contextuales como "Inspector de patio" o "Inspector de pasillo"; por último dentro del grupo directivo, al coordinador docente la mayoría habla del "Jefe UTP", salvo en el M1 en que se habla de "jefe docente". Respecto al profesor a cargo de un curso, la totalidad de los establecimientos municipales se refieren a él como "Profesor jefe".

Ahora bien, dentro de los establecimientos particulares subvencionados en 9 de 10 instituciones se habla de "director" o "directora", salvo en el PS3 que se utiliza el término "Rector"; frente al coordinador docente, en todos los establecimientos se habla de "Jefe UTP"; y, también en la totalidad de los establecimientos, se habla del "Inspector", con variantes como "Inspector de zona", "Inspector de patio" e "Inspector de pasillo". Respecto al profesor a cargo de un curso, la mayoría habla de "Profesor jefe", siendo la distinción, nuevamente en PS3 cuyo informante refiere a "Profesor guía". Dentro de los colegios católicos particulares subvencionados (PS11, PS12, PS13, PS14, PS15), la totalidad habla de "Profesor jefe" e "Inspector", aunque existen variantes en cuanto a la autoridad máxima: en PS11, PS14 y PS15 los estudiantes 
señalan el término "Directora"; en PS12, "Padre Director"; y en PS13 "Directora" o "Madre".

Finalmente, los cuatro estudiantes de colegios particulares pagados representan a los cuatro establecimientos de dicha dependencia en Talca. Frente a la autoridad máxima, en tres establecimientos se habla de "Rector" o "Rectora" y en el PP4 de "Directora", aunque también el apelativo de "Dueña" o "Sostenedora"; respecto al coordinador de profesores, los informantes de PP1, PP2 y PP4 hablan de "Jefe UTP", salvo en PP3 que refiere a "Coordinador Académico"; además, la totalidad de los particulares pagados utilizan el término "Inspector" con variantes como "Inspector de ciclo". Por último, todos los establecimientos hablan de "Profesor jefe".

\subsection{Denominación estudiante a profesor; profesor a estudiante}

En general todos los estudiantes de establecimientos municipales se refieren a sus docentes como "Profesores" y, en un buen número, también con el apócope "Profe", "tío"/"tía" y, en menor medida, "señorita". Además, la mitad de los establecimientos municipales se refiere como "Miss" a la profesora de inglés.

La totalidad de los estudiantes de establecimientos particulares subvencionados denominan "Profesores" a sus docentes, la mayoría también como "Profe" y "tío"/"tía". En el PS3, se refieren a algunos docentes como "Seño". Similares resultados se encuentran en colegios católicos, con la distinción que también se usa el apelativo de "Madre" o "Sor".

Los mismos resultados se encuentran en establecimientos particulares pagados, salvo en PP1 que subrayan la denominación de "Señor" o "Señora".

Considerando, por su parte, las denominaciones y apelativos utilizados por los docentes para referirse/dirigirse a los estudiantes, en los establecimientos municipales se utiliza el nombre o el apellido del educando; en general, también se usan términos como "joven", algunos diminutivos $\mathrm{y}$, en menor medida, a través de sobrenombres; y en los establecimientos M2, M8 y M9, algunos docentes los mencionan a través de los números de lista. Cuando los docentes se dirigen a todo el curso, suelen utilizar el término "alumnos", aunque también existen momentos particulares en que se habla de "niños", "pupilas", "muchachos" y "estudiantes".

A su vez, en los establecimientos particulares subvencionados, se repite la tendencia a que en todos se usa tanto los nombres como los apellidos, aunque en PS 3 y PS7 algunos docentes los llaman a través de un diminutivo, en PS5 como "Señor" o "Señorita", PS2 como "Hijo" o "Hija" y en PS6 y PS9 como "Joven". A su vez, cuando el docente requiere hablar a todo el curso se usa indiscriminadamente en todos los establecimientos: "Alumnos" o "Estudiantes" y en menor medida, "niños". Los resultados son similares en particulares subvencionados católicos.

Respecto a los establecimientos particulares pagados, los resultados no son muy distintos. Los cuatro estudiantes de los cuatro establecimientos particulares pagados refieren a que los docentes se dirigen a ellos a través del nombre o el apellido, aunque en PP1, PP2 y PP3 también se usan diminutivos, en PP2 y PP3, algunos docentes a través de sobrenombres $\mathrm{y}$, un docente en PP1, por el número de lista. Por último, cuando los docentes se dirigen al curso en general, usan tanto "alumnos" como "estudiantes", "niños" y "jóvenes".

\subsection{Denominación sobre aspectos disciplinares y estados de matrícula}


Todos los estudiantes de todas las dependencias escolares coinciden en que la terminología referida en esta categoría, para referirse a estados disciplinares resolutivos a nivel de establecimiento, es la siguiente: "Anotación Positiva", "Anotación Negativa", "Condicional", "Expulsión” y "Cancelación de Matrícula".

\subsection{Declaraciones de los profesores frente a actitudes y resultados negativos}

Dentro de los establecimientos municipales, los estudiantes señalan que en M1, M6, M8 y M9, frente a las malas calificaciones, siempre los comparan con otros cursos. Dichas asimilaciones se traducen en baja autoestima en los estudiantes y poca valoración por su trabajo, así lo menciona un informante:

M1: "Nos dicen: yo encontraba que este curso iba a ser mejor, porque estas cosas no ocurren en otro séptimo".

De hecho, el estudiante asume, que los docentes expresan lo mismo en otros cursos, utilizándolo como estrategia para fomentar la competitividad entre cursos. Lo anterior no es un episodio aislado. Lo reiteran la mayoría de los entrevistados:

M9: "Nos dicen que nos parecemos a otro curso, que somos el peor curso del colegio". Por su parte, los informantes mencionan ser objetos de retos por parte de sus profesores, ya sea por su mal comportamiento o por las bajas calificaciones, generando en los alumnos inseguridad y desmotivación. De esta manera, los estudiantes de M1, M5 y M7, aseguran ser víctimas de retos cuando evidencian un mal comportamiento, y en los M1 y M2, frente al bajo rendimiento. Es así como, la permanente acción de retar a los estudiantes -como ellos mismos lo afirman- predispone sus sentimientos y sensaciones frente a los docentes:

M1: "Los más pesados nos tratan peor".

Por otro lado, el informante del M10, manifiesta ser receptor de amenazas, que aluden a que no se les cambiarán las notas a final de año, porque no demuestran interés, lo que genera en los estudiantes sentimientos de rechazo y bajo interés frente a sus clases, como se puede evidenciar:

M10: "Nos amenazan con que a final de año cuando andemos pidiendo ayuda para pasar de curso nadie nos va ayudar."

Por el contrario, en M3, M4 y M6, se les incita a los alumnos a superarse frente a las bajas notas y mala conducta. Lo anterior lo subraya un informante:

M1:"que esperan algo mejor de nosotros".

Por su parte, en el M4 llama la atención la actitud de los docentes, pues el informante declara:

M4:"Nos trata de subir el ánimo siempre. Que podemos dar más. No nos retan mucho".

Dentro de los establecimientos de dependencia particular subvencionada, en PS1, PS2, PS3, PS5, PS6, PS9 y PS10, los informantes señalan que experimentan retos debido a las malas calificaciones. De hecho, en PS4, PS7, PS8 y PS9 indican que reciben retos, anotaciones negativas y además amenazas dentro del establecimiento por el mal comportamiento o las bajas calificaciones:

PS4: "Nos dice que no vamos a poder ir a ningún liceo bueno, porque tenemos muchas anotaciones y no vamos a poder estudiar."

Asimismo ocurre en PS5, en donde los profesores agregan la posibilidad de expulsión a los alumnos: PS5: “(...) que tenemos que cambiar, porque nos van a cancelar la matrícula para el próximo año, porque hay muchos niños esperando (...) que nos va a costar mucho encontrar un colegio tan bueno como éste." 
$\mathrm{Y}$ algo similar ocurre en PS6 en el cual el informante señala, en relación a las declaraciones de sus profesores:

PS6: "les va ir mal y yo a final de año no voy andar regalando décimas, ni tampoco voy a atender a los papás, y las lágrimas no me conmueven".

PS8: "A veces los amenazan con que los van a echar del colegio, o con la anotación negativa"

Por su parte, el informante dePS9, al igual que en un establecimiento municipal, señala que los comparan con otros cursos:

PS9: "Nos comparan con otros cursos, dicen que ellos son mejores que nosotros, y que no tienen estas notas".

Sin embargo, en el PS4 el informante indica que cuando es un estudiante quien obtiene bajas calificaciones, el trato es bastante positivo:

PS4: "Cuando por ejemplo es un alumno no más... los profes conversan con él y le dicen que él puede más, que tiene que mejorar".

De los colegios particulares pagados, los informantes dePP1, PP3 yPP4 manifiestan ser objetos de retos, cuando obtienen un bajo rendimiento.

$\mathrm{Al}$ informante de PP2, siempre lo retan por su mal comportamiento. Además, en este mismo establecimiento, se hacen comparaciones entre PP1 y PP2 y con el resto de los estudiantes del colegio. PP1: "Antes, decían que éramos más malos que el otro curso."

En el ámbito de las calificaciones, el informante de PP3 declara:

"los retan mucho y les dicen que no van a llegar a ningún lado, que estudien, que no sean flojos".PP4: "estoy muy avergonzada de ustedes, estudien más" (...) "los profesores dicen que somos lo peor del mundo, y que no servimos para nada, que nunca vamos a llegar a ser doctores y abogados."

\subsection{Declaraciones del profesor frente a actitudes y resultados positivos}

En los establecimientos municipales M1, M2, M3, M8 y M10, los felicitan cuando alcanzan logros u obtienen buenas calificaciones, sin embargo en M1 nunca es con palabras de admiración o muy elogiosas, subrayando que han cumplido y restando, en parte, los méritos. El informante reflexiona ante esto, aludiendo que es su deber portarse bien, entonces por eso los profesores no lo valoran más de la cuenta, incluso menciona:

M1: "Cuando nos portamos bien no nos felicitan. De repente es: muy bien. Pero nunca es con asombro" (...) "Hay una profesora que es como la más simpática y que ella siempre se fija en las cosas buenas que hacemos."

Asimismo, en los establecimientosM2, M4, M9 y M10, los profesores alientan a sus estudiantes expresándoles que debencontinuar así y, además, en M9, los animan para que puedan llegar a un buen liceo. En M5 y M9 les enuncian que van a registrar anotaciones positivas para que realicen alguna actividad o cuando ya la ejecutaron de manera eficiente. Por su parte, en M2, los docentes se muestran contentos y en M7, cuando realizan algo positivo, los congratulan delante de todo el curso y además felicitan a los papás en la reunión de apoderados. Una singularidad en este aparte es que el informante de M6 indica que nunca les dicen algo positivo.

En los establecimientos particulares subvencionados, los informantes dePS1 y PS3 señalan que regularmente los docentes indican que "están bien" los estudiantes.Además en los establecimientos PS2, PS5, PS6, PS7, PS8, PS9 y PS10, los docentes los felicitan 
cuando realizan acciones positivas u obtienen buenas calificaciones, pero además en PS3 no sólo el profesor lo reconoce, sino también el rector del establecimiento. Además, cabe agregar, que los docentes de PS1, PS4, PS7 y PS9, alientan a sus estudiantes a seguir así, los felicitan cuando obtienen buenas calificaciones y los instan a superarse. Lo anterior se refleja en lo manifestado por el informante PS2:

"Nos felicitan y nos dicen que tenemos que estudiar pa' la otra prueba igual, porque nos tiene que ir bien igual que la otra. Y cuando a unos pocos les va bien, dicen que tiene que ser todo el curso igual, que si uno puede, el otro igual puede."

PS1: "Nos dicen que está súper bien y que sigamos así."

PS4: "En consejo de curso nos dicen que somos el mejor curso y que tenemos que seguir así, porque vamos a poder elegir un buen colegio y no el colegio a nosotros".

Asimismo, el informante dePS9 afirma:

"Cuando me va bien, me felicitan y me preguntan si estudié, y yo les digo que sí, entonces me dicen que siga así, y va a obtener buenos resultados."

En el mismo sentido se traduce lo que menciona el informante dePS10:

"te felicitan, que te puedes superar aún más, como que siempre te dan más apoyo."

Cabe señalar que en PS13, colegio católico, les dan las gracias públicamente por participar en la pastoral.

Finalmente, en los establecimientos particulares pagados, los informantes de PP1, PP2 y PP4 señalan que los profesores los felicitan, pero sólo felicitan a los más descendidos para que suban sus notas. Se subraya que en todos los alientan a seguir así, valorando los logros de sus estudiantes:

PP4: "Nos dicen que sigamos así, que está bien, que somos inteligentes y que tenemos que rendir". Por su parte en PP2 un profesor bromea con los estudiantes preguntándoles si han copiado y reforzando el trabajo que realizan con todos los docentes.

2. Comprender las percepciones y creencias de los estudiantes acerca de la identidad lingüística de los establecimientos escolares urbanos de la ciudad de Talca

\subsection{Declaraciones del profesor gratas para el estudiante}

Dentro de los establecimientos municipales existen algunas coincidencias que es importante resaltar. Por ejemplo, los informantes de M1, M3, M5, M6 y M8 valoran que se les llame por su nombre, por sobre el apellido u otros apelativos:

M5: "Me gusta que me llamen por mi nombre porque no se saben todos"

M6: "Me agrada que me llamen por el nombre, porque es como más cercano"

M8: "Me da igual, me gusta que me llamen por mi nombre".

Por otro lado, las felicitaciones de los docentes son muy bien recibidas y valoradas por los estudiantes:

M1: "Me gusta cuando me felicita, cuando dicen que somos un curso bueno, unido".

M2: "Me gusta que me digan que estoy bien, que me superado que todo, que he mejorado pa' bien o pa mal po'. Ayer po', ayer me felicitaron porque yo tenía puras buenas notas".

M3: "Me gusta que me feliciten (sonríe) es un agrado".

Además, M4 valora mucho el sentido del humor, cuando la profesora actúa con sana ironía, "haciéndose la tonta" o la que no entiende o juega que los estudiantes no la tratan bien. Por supuesto que también se encuentran particularidades como el informante de 
M9, que refiere lo positivo que es que hablen bien del curso en los consejos de profesores o, como indica el informante de M7, cuando les dicen que no hay clases o, incluso, como señala el estudiante de M10, cuando les avisan que cambiarán la prueba. Para el educando de M8, el hecho que los profesores los incentiven, alaguen y apoyen es clave:

"Nos sentimos bien cuando la profe jefe siempre dice que podemos mejorar y cosas así, y la mayoría de los profesores nos trata de motivar".

Un resultado medianamente similar se obtiene en los establecimientos particulares subvencionados. También destacan las felicitaciones, siempre bien recibidas y valoradas por los estudiantes:

PS2: "Me gusta que me feliciten, me encanta, me siento como que logré algo".

PS3: "Cuando nos felicitan, sí. Eso me gusta"

PS7: "Cuando me felicitan por mis buenas notas en consejo de curso y todas se enteran, y me siento súper orgulloso".

PS11: "Me gusta que me feliciten cuando me va bien en las pruebas, que reconozcan el esfuerzo que uno hace para que le vaya bien".

De igual manera, algunos informantes subrayan la importancia que se les salude, como es el caso de los informantes de PS1 y PS3. En el caso de esta dependencia, sólo un estudiante (PS10) hizo referencia a sus sentimientos positivos cuando se les llama por su nombre. A su vez, dos informantes (PS4 y PS7) valoran muchísimo los reconocimientos públicos que se hacen de los logros personales:

PS4: "Me gusta cuando dicen los nombres de los que tienen mejores notas, los usan de ejemplos y que seamos como ellos, y este año me han nombrado harto por la superación y el esfuerzo y me siento súper bien".

Por supuesto que también reciben de manera muy positiva cuando les avisan de cambios en el contexto de clases (salir al patio, ir a la sala de computación) o cuando se les compromete con un refuerzo positivo (salir antes a recreo, subir la nota, regalar unas décimas).

Finalmente, en los establecimientos particulares pagados, los informantes de PP1 y PP2, indican que se sienten bien cuando se les felicita, tanto de manera particular como general. El estudiante de PP3, a su vez, se siente agradado cuando se les prometen décimas para la prueba y en PP4, cuando le dicen "Señor", porque siente que se le habla con respeto.

\subsection{Declaraciones del profesor ingratas para el estudiante}

Dentro de los establecimientos municipales, las percepciones son variadas y subrayan lo referido en la categoríaDeclaraciones de los profesores frente a actitudes y resultados negativos de los estudiantes. Para el informante de M1, no es grato que los profesores les llamen la atención para que los nombren como "Profesor", "Director" o "Inspector", subrayando la autoridad que connota. En M2, a su vez, el entrevistado indica que no le "gusta que le corrijan de mala forma" y, tanto en M3, M8 y M10, lamentan que los docentes los comparen con otros cursos, generaciones o colegios:

M10: "Cuando nos dicen que somos el peor octavo que ha salido del colegio".

También existe consenso en rechazar los sobrenombres sobre los estudiantes que son expresados por los docentes. En ese punto, el informante de M2 es enfático: 
"Algunos se tratan con sobrenombres, cabezón, negro pero a mí no me dicen sobrenombres, eso no me gusta".

Varios informantes, además, puntualizan el desagrado que les provoca el hecho que los docentes subrayen su autoridad y manejo del conocimiento, por sobre los educandos:

M6: "Los profesores dicen que ellos tienen la autoridad y pueden hacer lo que se les ocurra en sus clases. Se creen perfectos nunca se equivocan, siempre tienen la razón."

M1: "Hay cosas que se hacen todo lo contrario. Por ejemplo, ayer creo que fue, nunca había pasado, pero... eeeh, siempre los profesores dicen que no faltemos el respeto, pero eeeh, ayer una profesora nos faltó el respeto: dijo que éramos unos limitados y que ella sabía lo que hacía".

El resto de los informantes indican sentimientos de rechazo ante declaraciones que apuntan a denominaciones, incentivos o retos:

M4: "No me gusta que nos traten de niños es como si nosotros los tratáramos de viejos a ellos".

M5: "No me gusta que me llamen por mi apellido porque es como rasca".

M6: "No me gusta que nos reten por tonteras, porque nos paremos a botar un papel al basurero nos retan... nos dicen ¿a qué te paraste?"

M9: "Que tenemos que hacer trabajos con notas porque si es sin nota no hacemos nada".

Considerando los establecimientos particulares subvencionados, las percepciones de los informantes van por la misma línea. En primer lugar, también surgen sentimientos negativos frente a las comparaciones. Para el informante de PS4 es muy molesto que los comparen con los otros octavos e incluso algunos docentes señalan que los otros cursos llegarán a determinados liceos y ellos no. En el caso del entrevistado de PS10, sucede algo similar, ya querefiere que aunque se les considera mejores, siempre está la advertencia que pueden ser superados:

PS10: "Nos comparan, siempre con el octavo B, que nosotros somos mejores, pero si 'tamos así terminaremos peor que el otro curso y cosas así".

También algunos informantes son claros en comentar que les desagrada que los reten o que los amenacen con anotaciones negativas o que, finalmente, les adviertan y quiten minutos del recreo:

PS1: "No me gusta que nos reten, porque a veces no es culpa de todos, no estamos haciendo todo desorden y nos ponen una anotación general".

Además, indican que les molesta que declaren una cosa y luego actúen de una manera incoherente:

PS11: "Las monjas siempre dicen que no hay que usar tanto teléfono o Tablet, que nos podemos marear o atrofiar el cerebro y ellas mismas andan con los medios teléfonos, con las medias cámaras."

PS14: "Los profesores son raros, porque andan hablando de Dios, y cuando le hacemos preguntas no nos responden, gritan mucho, llegan tarde a la sala, y nos retan por puras tonteras".

Un par de informantes (PS2 y PS13) señalan que les incomoda y molesta que les digan hijas y que prefieren que se les llame por su nombre, tanto así que el estudiante de PS11 indica:

"Con la inspectora yo llevo seis años y nunca se ha aprendido mi nombre". 
En general en los establecimientos particulares pagados están más conformes con las emisiones lingüísticas de los docentes. En PP4 el estudiante precisa que no le gusta cuando les advierten que con esa actitud no llegarán a ningún lado y en PP1, se puntualiza que no le gusta que le llamen por su nombre completo.

\subsection{Términos utilizados dentro del establecimiento que el alumno cambiaría}

En los establecimientos municipales destaca que la mayoría de los informantes preferirían dirigirse o referirse a los profesores por su nombre, incluso a directivos, por ejemplo:

M1: "Todos deberíamos tratarnos por el nombre. Podríamos agregar el don."

M2: "Me gustaría llamar por el nombre a los profesores".

M5: "Puede ser que a los profesores se les llamara por su nombre".

Frente a otras instancias del proceso educativo, existen algunas sugerencias de parte de los informantes. EL educando de M4 indica que sería mejor que a los auxiliares se les denominara de alguna manera más respetuosa; el informante de M9 propone que la sala de clases tome un nombre más entretenido, que invite a la diversión; y el estudiante de M10 puntualiza que las pruebas deberían denominarse como tareas o trabajos, ya que así "darían más ganas de estudiar". Cabe destacar que para el entrevistado de M3 nada debiera cambiar y para el educando de M8, no podría dirigirse a los profesores por sus nombres, pues a su juicio "se volvería chacota".

Mucho más variada es la posición de los informantes de los establecimientos particulares subvencionados, ya que existen pocas coincidencias y un amplio campo temático de opiniones. Por ejemplo, para los estudiantes de PS7, PS10 y PS13 la frase "Anotación Negativa" debiera modificarse por otra, que esté más relacionada con un objetivo didáctico; a su vez, para el alumno de PS3, no debiera usarse el término "suspendido" al igual que para el informante de PS9, que bromea:

"Sí, cambiaria suspensión, le pondría un día de vacaciones".

En cuanto a las denominaciones de los docentes, varios coinciden en que debieran realizarse cambios. Para el informante de PS1 lo ideal sería llamarles "Tíos" o "Tías" a todos; "Sostenedor", a su vez, es un término que el estudiante de PS5 cambiaría; y para el educando de PS6, el apelativo de "Profesor jefe" no debiera usarse:

"Es como que él manda en la sala, y no es así, él nos enseña".

Para el alumno de PS4 sería fundamental que las "Pruebas" tomaran otro nombre, pues la encuentra muy "fea" y para el estudiante de PS8, los auxiliares debieran denominarse de otra manera:

“Auxiliar, porque los tíos son simpáticos y los profes los tratan mal, les piden cosas, y los llaman despectivamente".

Dentro de los establecimientos católicos, los estudiantes señalan distintos cambios que realizarían, como, por ejemplo, llamar a los docentes por sus nombres, no denominarles "Padre Director" o "Padre" y menos "Inspectora".

Finalmente, dentro de los establecimientos particulares pagados sólo se puntualiza que no les gusta la denominación de "Rector" (PS2, PS3), consideran que el término "Auxiliar" es despectivo (PP1) y, para el estudiante de PP4 sería importante sólo que fueran un poco más sutiles para decir las cosas. 


\section{Discusión y reflexiones}

\section{Describir la identidad lingüística de los establecimientos escolares urbanos de la ciudad de Talca}

Los estudiantes de los establecimientos escolares urbanos de Talca participantes en el estudio, dan cuenta de una descripción lingüística bastante homogénea, sin mayores diferencias en torno a dependencias. En el caso de los equipos de gestión se reiteran los términos "Director", "Jefe de UTP", "Inspector" y, en menor medida, "Rector", "Jefe docente" o "Madre". En el caso del docente a cargo de un curso, es preponderante el uso de "Profesor jefe" por sobre, un caso, "Profesor guía". En ese sentido, se colige que, más allá de las prácticas y las actitudes, la identidad lingüística descrita en este punto, manifiesta una diferenciación jerárquica, siendo el funcionario o el docente "superior" al estudiante, sobre todo si nos centramos en el docente a cargo del curso, quien, finalmente, es el funcionario más cercano a los educandos, el cual sigue siendo denominado como "Profesor Jefe", no popularizándose aún un término más relacionado incluso con el curriculum, como es "Profesor guía". En el caso de "Director", si bien se connota su significación jerárquica, también se connota su significación funcional, lo cual no representaría una marcada relación asimétrica, a diferencia de "Rector" cuya presencia sí connota un mayor determinismo y control, término acuñado más que nada en sectores particulares de la educación escolar.

En cuanto a los apelativos utilizados por los docentes para dirigirse a los estudiantes, el panorama es muy similar por dependencia. La mayoría de los estudiantes coinciden que se les denomina por el nombre o el apellido. No obstante, llama la atención que algunos docentes se dirigen a ellos a través de sobrenombres y diminutivos, lo cual no significa que sea mal recibido por el estudiante y, en otros casos, a través de números de lista, lo cual sí posee una connotación negativa: la impersonalidad de esa práctica discursiva subraya, a decir de Van Dijk, la desigualdad entre unos y otros. De hecho, en parte, resta al carácter identitario de los receptores.

De acuerdo a los apelativos utilizados por los estudiantes para referirse a sus docentes, por otro lado, no se pesquisan mayores diferencias por dependencia. "Profesores", "Profe", "Señorita" y, en menor medida, "Seño", "Tío(a)", "Madre" son el denominador común en la manera que usan los estudiantes para dirigirse a sus docentes. Por tanto, se reproduce la histórica importancia del rol dentro de la sala de clases, manteniéndose el orden de relaciones regularmente establecido.

En cierto modo, la información de los estudiantes entrevistados refleja una reproducción en la escuela del contexto segregado dominante, bien documentado hoy en día sobre nuestro país. La importancia de las jerarquías, las denominaciones de cargos, las relaciones asimétricas de nuestra sociedad se intencionan en los establecimientos escolares no pesquisándose disonancias.

A su vez, en las denominaciones de aspectos disciplinares y estados de matrícula, la coincidencia es absoluta, al utilizarse en todas las dependencias los términos: "Anotación positiva", "Anotación negativa", "Condicional", "Expulsión" y "Cancelación de matrícula". En ese sentido, no se evidencia ninguna evolución lingüística en estos aspectos identitarios de la institucionalidad escolar, que podrían generar prácticas discursivas más acordes a un ambiente de encuentro y reflexión -que 
debiera ser más amable y grato para el estudiante- y menos emparentado con una terminología que coincide con el hábitat penitenciario.

Dentro de las declaraciones de los docentes frente a actitudes y resultados negativos de los estudiantes, se reiteran varias prácticas discursivas. Las comparaciones entre cursos y con otros establecimientos, fomentando la competitividad, suelen estar presentes en las escuelasde todas las dependencias, según declaran los estudiantes entrevistados; de tal manera, se va generando una baja autoestima en los educandos y se fortalece una competencia centrada en "ser mejor que otro" y no centrada en la autosuperación, el autoconcepto y la creación de expectativas. Por otro lado, los retos, e incluso amenazas de reprobación o expulsión, también son usuales, indican los estudiantes, creando un ambiente negativo dentro de las aulas. Naturalmente que, estas prácticas, generan, se insiste desde Mateos, una percepción negativa sobre la cultura escolar, lo cual aumenta las posibilidades de fracaso escolar y poca pertenencia a la identidad lingüística de la escuela. De igual manera, los estudiantes destacan que en ocasiones el discurso docente invita a la superación, a elevar la autoestima y a expresar altas expectativas para revertir escenarios de bajos resultados.

Ahora bien, de acuerdo a las declaraciones del profesor frente a actitudes y resultados positivos, las felicitaciones públicas en la sala suele ser la práctica habitual de los docentes, al igual que el instarlos a seguir así o el animarles a continuar en esa senda de esfuerzo y logros. Sin embargo, en ocasiones las emisiones lingüísticas se tiñen de incentivos externos al prometerles anotaciones positivas o al instalar que la motivación sería llegar a un buen liceo. En rigor, la mayoría de los entrevistados considera que ante los logros positivos reciben palabras pertinentes y gratas, con algunos bemoles, por ejemplo, en que los docentes no hacen mayores declaraciones frente a los éxitos, restando méritos al estudiantado, tal vez con la intención de no generar un exceso de confianza ante los resultados que pudiera provocar menor esfuerzo a futuro.

2. Comprender las percepciones y creencias de los estudiantes acerca de la identidad lingüística de los establecimientos escolares urbanos de la ciudad de Talca

Considerando las declaraciones del profesor que son bien recibidas por los estudiantes, los informantes declaran -en los tres tipos de dependencias- que perciben muy positivamente que los docentes les feliciten o hagan reconocimientos públicos sobre los resultados o les manifiesten altas expectativas o los inviten a la superación. Para los entrevistados provenientes de establecimientos municipales, es muy importante que se les llame por su nombre, aquello genera cercanía, por sobre el uso de apellidos u otros apelativos, así como para algunos informantes de particulares subvencionados es bien valorado que se les salude.

Naturalmente que el fortalecimiento de una identidad lingüística más propia de decisiones discursivas positivas (felicitar, alagar, declarar expectativas) desarrollará en los estudiantes un fortalecimiento del vínculo con la comunidad de habla (Martínez) y fortaleciendo el sentido de pertenencia (Molano). En ese sentido, se insiste, mientras el estudiante se sienta más cómodo y satisfecho en su establecimiento escolar, envuelto en un discurso de cercanía, reconocimiento y atención, tenderá a obtener mejores resultados.

Por otro lado, las prácticas discursivas de autoritarismo, comparación, retos e incoherencias con la acción, resultan ser los aspectos de la identidad lingüística que más desagradan a los estudiantes de las tres dependencias. En ese sentido, no se puede negar 
que en toda institución educativa es natural que surjan discrepancias, desavenencias y discusiones en el complejo tejido de la interacción docente-estudiante. Las frustraciones de uno u otro, las representaciones y los modelos mentales de cada interlocutor pueden originar discursos negativos, incluso ofensivos, cuando se alejan de los moldes preestablecidos, no se cumplen los objetivos o no se alcanzan las expectativas. No obstante, cabe acá la reflexión en torno a si las decisiones lingüísticas que se toman en estos casos, pueden orientarse desde una intención pedagógica que, en vez de generar percepciones negativas de los estudiantes, permita desarrollar la autoevaluación, autocrítica y, sobre todo, instalar la idea de aprender del error, en el sentido de Saturnino de la Torre (2004). Es decir, que aquello que es considerado como negativo por el profesor, no se transforme en un reactivo de retos y comparaciones antojadizas, sino en una oportunidad de formación. En esa línea, cuando el docente compara al grupo de estudiantes con otros cursos o compañeros, declaran los educandos que sólo genera rabia o frustración y no el esperado sentido de superación. Se podría colegir, por último, que los estudiantes no reciben con agrado el fomento de la competencia por lo menos a esta edad, ya que resulta evidente que si la práctica discursiva persiste, provocará efectos ideológicos importantes en los receptores, reproduciendo un contexto de competitividad y desigualdad, desde la perspectiva de Teun Van Dijk.

Por último, los estudiantes de las tres dependencias cambiarían aspectos ya discutidos como llamar por el nombre a los funcionarios del sistema educativo, tal vez con la intención de generar relaciones más asimétricas en desmedro de la jerarquía y el autoritarismo. Aparentemente, los informantes valoran la cercanía que se manifiesta a través de la identidad lingüística. De tal manera, también se insiste en la idea de no reproducir un contexto de dominación con terminologías como "Anotación negativa", "Suspensión" o "Condicionalidad", ya que son recibidas con desagrado por los estudiantes.

\section{Conclusiones}

Múltiples son las conclusiones que se pueden sacar de este estudio y, por sobre todo, innumerables las profundizaciones investigativas que podrían complementar de mejor manera esta breve exploración a la identidad lingüística escolar; toda vez que esta investigación no ha buscado la representatividad ni la generalización a todo el contexto educativo, sino más bien el rescatar la visión subjetiva de algunos estudiantes, recogiendo información clave que entrega señales sobre la cultura de las escuelas.

En primer lugar, los estudiantes describen una identidad lingüística que posee reminiscencias de autoritarismo y jerarquías bien distinguidas, considerando relaciones asimétricas entre los funcionarios y los estudiantes, aspecto que los informantes declaran percibir de manera negativa. "Profesor Jefe", "Rector", "Inspector", "Condicionalidad" son términos que podrían analizarse en cuánto al rol que están desempeñando en el contexto escolar -de qué manera, por ejemplo, reproduce un contexto social complejo- y, evidentemente, las representaciones mentales que generan en los estudiantes.

En segundo lugar, en la sala de clases los estudiantes reciben positivamente todas aquellas emisiones lingüísticas que se orientan a la felicitación, la cercanía y la superación, sintiéndose más involucrados y comprometidos con el proceso educativo y la institución. 
En tercer lugar, por el contrario, los informantes rechazan la regular ocurrencia de prácticas discursivas que comparan con otros, amenazan o retan los fracasos o actitudes disciplinarias negativas de los estudiantes. En ese sentido, sería adecuado crear una cultura escolar que tome el error no como un motivo de castigo verbal, si no como una oportunidad más de aprendizaje.

En consideración con lo anterior y destacando que no se recogen diferencias mayores entre las dependencias administrativas, cabe destacar que las declaraciones de los estudiantes entrevistados evidencian que a medida que la identidad lingüística tiende a emisiones positivas, simétricas y cercanas, su compromiso y sentido de pertenencia con el establecimiento tiende a ser mayor, al igual que su esfuerzo para alcanzar mejores resultados. En cambio, una identidad lingüística más distante en jerarquías, autoritaria y con un discurso más adoctrinador, competitivo y agresor verbalmente, trae como consecuencia un rechazo del contexto escolar por parte de los informantes.

Se sugiere, por último, el reflexionar y revisar la identidad lingüística propia de los establecimientos escolares, de tal manera que se evalúen las prácticas discursivas a la luz de una intención pedagógica eficiente, integradora y más simétrica, de tal forma que los estudiantes forjen vínculos afectivos con la comunidad hablante institucional, fortaleciendo el sentido de pertenencia y, en definitiva, los compromisos y resultados de aprendizaje.

\section{BIBLIOGRAFÍA}

1. Agulló, E. (1997). Jóvenes, trabajo e identidad. Oviedo, España: Universidad de Oviedo.

2. Alarcón, R. (1990). Identidad de la psiquiatría latinoamericana: voces y exploraciones en torno a una ciencia solidaria. México D. F, México: Siglo veintiuno editores S.A.

3. Araya, C. (2011) Análisis crítico de tres afiches de campaña contra la violencia hacia la mujer: maricón es el que maltrata a una mujer. Contextos, $\mathrm{N}^{\mathrm{o}} 25$, pp.1328.

4. Mateos, T. (2008). La percepción del contexto escolar. Una imagen construida a partir de las experiencias de los alumnos. Cuestiones pedagógicas, $\mathrm{N}^{\circ} 19$, pp. 285 300

5. De la Torres, S. (2004). Aprender de los errores. El tratamiento didáctico de los errores como estrategia de innovación. Buenos Aires, Argentina: Editorial Magisterio del Río de la Plata.

6. Díaz, E. (2009). Prácticas comunicativas e identidad. Santiago de Compostela, España: Universidad de Santiago de Compostela.

7. Gómez, V; Herrera, L. (2014) Idiosincrasia del valle central chileno: Diccionario de Neologismos y Locuciones Usuales. Papeles de trabajo, $\mathrm{N}^{\mathrm{o}} 28$, pp. 79-94

8. Herrera, L. (2014). Reflexión sobre los propósitos de la sociedad y la educación actual. Foro Educacional, No23, pp.85-100

9. Dahmen, W., Holtus, G., Kramer, J., Melzeltin, M., Schweickard, W., Winkelmann, O. (2006) Lengua, Historia e identidad. Perspectiva española e hispanoamericana. Alemania: Narr.

10. Martínez M. (2008). La identidad lingüística y los trastornos del habla. Boletín de lingüística, vol 20, $\mathrm{N}^{\circ} 29$, pp. 85-101 
11. Lozano, J.; Peña, C.; Abril, G. (1999) Análisis del discurso. Hacia una semiótica de la interacción textual. Madrid, España: Ediciones Cátedra S.A.

12. Monereo, C.; Pozo, J. (2011) La identidad en Psicología de la Educación: necesidad, utilidad y límites. Madrid, España: NARCEA S.A.

13. Molano, O. (2008). La identidad cultural, un concepto que evoluciona. Revista Ópera, $\mathrm{N}^{\circ} 7$, pp. 69-84.

14. Montoya, V. (2001) [En línea]Apuntes pedagógicos. Sincronía, $N^{\circ} 21$. Recuperado el 26 de Marzo 2015, de:http://www.espaciologopedico.com/revista/articulo/1434/la_escuela_como_refle jo de la sociedad.html

15. Stolp, S.; Smith, S. (1994) School Culture and Climate: The Role of the Leader. OSSCBulletin. Eugene: OregonSchoolStudy Council.

16. Van Dijk, T. A. (2012). Discurso y Contexto, un enfoque sociocognitivo. Barcelona, España: Gedisa, S.A.

17. Van Dijk, T. (2008). El discurso como interacción social. Estudios sobre el discurso II. Una introducción multidisciplinaria. Barcelona, España: Gedisa Editorial. 


\section{ANEXO 1}

Nombre:

Edad:

Colegio:

Curso:

Dependencia del colegio: Municipal

Particular Subvencionado

Particular Pagado

\section{Entrevista}

\section{Indicaciones:}

Se realizará una entrevista semi-estructurada. A partir de las preguntas ya estipuladas y de las respuestas de los entrevistados, se procederá, de ser necesario, a algunas contrapreguntas. Lo anterior, con el objeto de extraer mayor información y, a su vez, de explorar áreas importantes, que no habían sido consideradas.

\section{Objetivo general:}

$\checkmark$ Analizar la identidad lingüística de los establecimientos escolares urbanos de la ciudad de Talca, identificando su relación con la cultura escolar.

\section{Objetivos específicos y preguntas}

\section{$\checkmark$ Describir la identidad lingüística de los establecimientos escolares urbanos de la ciudad de Talca.}

1. Refiérase si se utilizan los siguientes términos dentro de su establecimiento: Rector, Director, Jefe de UTP, Inspector general, Profesor jefe. ¿Se utilizan otros? ¿Cuáles?

2. Comente de qué manera se dirige a los docentes. ¿Utiliza los términos Señor (a), tío (a), profesor (a), por su nombre, por su apellido, profe, etc.?

3. Explique la manera como se dirigen los profesores hacia sus alumnos: por su nombre, por su apellido, por su número de lista, como alumno, estudiante, por algún sobrenombre, etc.

4. ¿Utilizan los términos anotación positiva, negativa, citación de apoderado, suspensión, condicionalidad, expulsión? ¿Algunos otros? ¿Cuáles? 
Centro de Estudios Interdisciplinarios en Etnolingüistica y Antropología Socio-Cultural

5. Describa qué dicen los profesores, para reforzar los logros o actitudes positivas de los alumnos.

6. Describa qué dicen los profesores, frente a malas calificaciones, errores o actitudes negativas de los alumnos.

7. ¿Qué otros nombres, palabras, dichos son recurrentes en los profesores, directivos, estudiantes del establecimiento?

$\checkmark$ Comprender las percepciones y creencias de los estudiantes acerca de la identidad lingüística en los establecimientos escolares urbanos de la ciudad de Talca.

1. ¿Qué nombres, palabras o dichos que expresan los profesores o estudiantes le agradan? ¿Por qué?

2. ¿Qué nombres, palabras o dichos que expresan los profesores o estudiantes le desagradan? ¿Por qué?

3. ¿Cambiaría algunas palabras utilizada frecuentemente dentro de su establecimiento? ¿Por qué? ¿Por cuál?

4. ¿Cómo te sientes frente a las denominaciones que recaen sobre ti en el establecimiento? ¿Por qué? 


\begin{abstract}
ANEXO 2

\section{Consentimiento informado}

He aceptado participar como entrevistado en la investigación de la Facultad de Educación de la Universidad Autónoma de Chile "Identidad lingüística del contexto escolar" desarrollada por los docentes Luis Herrera, Paula Espinoza, Katterine Flores, Yoselin Iturriaga y Javiera Poblete.

La entrevista se realiza en un lugar y hora convenida con los investigadores. Su duración es de aproximadamente unos 45 minutos. Durante la entrevista puedo hacer preguntas o hacer intervenciones y tengo el derecho a terminarla cuando yo lo estime conveniente.

Mi participación es voluntaria y no involucra compensación. Entiendo que mi nombre, y lo que diré en esta entrevista, es confidencial. Esto significa que si los resultados de esta investigación son publicados, mis datos aparecerán de forma anónima. Podré conocer los resultados de esta investigación si así lo deseo, ya que la publicación, en caso de realizarse, será de acceso público.

Una copia de esta carta de consentimiento quedará para mí y la otra copia para el investigador.

Al aceptar participar en esta investigación autorizo la publicación de la información proporcionada en informes, publicaciones y revistas científicas.
\end{abstract}

Nombre participante:

Firma:

Entrevistador: Firma:

Lugar (comuna/ciudad): Talca. Fecha: 


\section{ANEXO 3}

\begin{tabular}{|c|c|}
\hline \multicolumn{2}{|c|}{$\begin{array}{l}\text { Describir la identidad lingüística de los establecimientos escolares urbanos de la } \\
\text { ciudad de Talca }\end{array}$} \\
\hline CATEGORÍA & GLOSA \\
\hline $\begin{array}{l}\text { Denominación de } \\
\text { funcionarios. }\end{array}$ & $\begin{array}{l}\text { Reúne los términos que deben utilizar los estudiantes para } \\
\text { referirse/dirigirse a los funcionarios que forman parte del } \\
\text { establecimiento educacional. }\end{array}$ \\
\hline $\begin{array}{l}\text { Denominación profesor } \\
\text { a estudiante; } \\
\text { estudiantes a profesor. }\end{array}$ & $\begin{array}{l}\text { Reúne los términos que los estudiantes deben utilizar para } \\
\text { referirse/dirigirsea los docentes y las denominaciones que } \\
\text { usan los docentes para referirse/dirigirse a los estudiantes. }\end{array}$ \\
\hline $\begin{array}{l}\text { Denominación sobre } \\
\text { aspectos disciplinares y } \\
\text { estados de matrícula. }\end{array}$ & $\begin{array}{l}\text { Reúne los términos que emplean los integrantes } \\
\text { administrativos y docentes para calificar las conductas de los } \\
\text { estudiantes, relacionados con temas disciplinares. }\end{array}$ \\
\hline $\begin{array}{l}\text { Declaraciones de los } \\
\text { profesores frente a } \\
\text { actitudes y resultados } \\
\text { negativos }\end{array}$ & $\begin{array}{l}\text { Reúne las declaraciones de los profesores que son negativas } \\
\text { sobre el curso, generalmente frente al aprendizaje, } \\
\text { rendimiento y la disciplina en la sala. }\end{array}$ \\
\hline $\begin{array}{l}\text { Declaraciones del } \\
\text { profesor frente a } \\
\text { actitudes y resultados } \\
\text { positivos }\end{array}$ & $\begin{array}{l}\text { Reúne las declaraciones de los profesores que son positivas } \\
\text { sobre el curso, generalmente frente al aprendizaje, } \\
\text { rendimiento y la disciplina en la sala. }\end{array}$ \\
\hline \multicolumn{2}{|c|}{$\begin{array}{l}\text { Comprender las percepciones y creencias de los estudiantes acerca de la identidad } \\
\text { lingüística de los establecimientos escolares urbanos de la ciudad de Talca }\end{array}$} \\
\hline $\begin{array}{l}\text { Declaraciones del } \\
\text { profesor gratas para el } \\
\text { estudiante }\end{array}$ & $\begin{array}{l}\text { Declaraciones y términos que expresan los docentes y que } \\
\text { son recibidas con agrado por los estudiantes. }\end{array}$ \\
\hline $\begin{array}{l}\text { Declaraciones del } \\
\text { profesor ingratas para } \\
\text { el estudiante }\end{array}$ & $\begin{array}{l}\text { Declaraciones y términos que expresan los docentes y que } \\
\text { son recibidas con desagrado por los estudiantes. }\end{array}$ \\
\hline $\begin{array}{l}\text { Términos utilizados } \\
\text { dentro del } \\
\text { establecimiento que el } \\
\text { alumno cambiaría }\end{array}$ & $\begin{array}{llcllrr}\text { Declaraciones } & \text { y } & \text { términos } & \text { que se expresan en } & \text { el } \\
\text { establecimiento } & \text { y } & \text { que los } & \text { estudiantes modificarían } & \text { o } \\
\text { anularían. } & & & & & & \end{array}$ \\
\hline
\end{tabular}

